\title{
Vascular Ring in an Infant With Cystic Fibrosis
}

\author{
Justin T. Tretter ${ }^{\mathrm{a}, \mathrm{b}}$, Puneet Bhatla ${ }^{\mathrm{a}}$, Sujata Chakravarti ${ }^{\mathrm{a}}$
}

\begin{abstract}
A vascular ring (VR) is a rare congenital anomaly that has variable clinical presentation, but may cause trachea-esophageal compressive symptoms. We describe an infant with cystic fibrosis and subtle trachea-esophageal compressive symptoms which were initially attributed to other causes, delaying the diagnosis of a VR.
\end{abstract}

Keywords: Vascular ring; Right aortic arch; Aberrant left subclavian artery; Cystic fibrosis

\section{Introduction}

Vascular ring (VR) is a relatively rare aortic arch anomaly representing $1-3 \%$ of congenital heart disease [1]. Clinical presentation varies from asymptomatic to compressive trachea-esophageal symptoms which can present as early as infancy to as late as adulthood. Due to the rare occurrence of VR with often insidious symptoms, diagnosis is often delayed and initially attributed to more common causes of similar symptoms [2, 3]. We report the case of an infant with cystic fibrosis (CF), in whom subtle compressive trachea-esophageal symptoms were initially attributed to CF causing delayed diagnosis of VR.

\section{Case Report}

The patient is a 4-month-old ex-36-week Hispanic twin male with $\mathrm{CF}$ and failure-to-thrive who presented to the emergency room with a 2-day history of choking with each feed,

Manuscript accepted for publication December 22, 2014

aDivision of Pediatric Cardiology, New York University Medical Center, New York, NY, USA

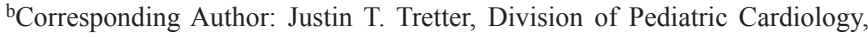
New York University Medical Center, 160 East 32nd St., L-3 Medical, New York, NY 10016, USA. Email: Justin.Tretter@nyumc.org

doi: http://dx.doi.org/10.14740/jicp178w one episode of non-bloody, non-bilious emesis and 2 days of worsened noisy breathing, but normal work of breathing between choking episodes. He had never had choking episodes before, but had an intermittent cough and occasional noisy breathing for 1 month. He had been afebrile with no nasal congestion or rhinorrhea, and no sick contacts.

His past medical history was significant for meconium aspiration at birth resulting in a 2-week hospitalization for respiratory distress and feeding issues. He had persistent poor weight gain, and was re-hospitalized at 1 month of age. During this admission, he was noted to have frequent copious yellow bowel movements along with anemia. He was transitioned to elemental formula with some improved feeding and weight gain. Pediatric pulmonology was consulted due to an elevated immunoreactive trypsinogen result on his New York State Newborn Screen with no CF mutations detected. Sweat testing confirmed $\mathrm{CF}$, and stool pancreatic elastase showed severe exocrine pancreatic insufficiency. He was started on pancreatic enzyme, salt and fat-soluble vitamin supplementations. He was noted to have an intermittent cough, and was empirically started on antibiotics pending deep pharyngeal cultures. These showed no bacterial growth and antibiotics were subsequently discontinued.

In the emergency room, his physical examination was significant for height and weight less than the fifth percentile for age. He was not in acute distress. While sleeping, he had normal work of breathing and respiratory rate with no adventitious breath sounds, but he had significant biphasic stridor and mild subcostal retractions while awake. He maintained normal oxygen saturations on room air. Chest X-ray was notable only for a right aortic arch (RAA). Bronchoscopy was performed for evaluation of the stridor, and demonstrated right-sided severe pulsatile tracheal compression. Esophagoscopy showed posterior pulsatile compression of the mid-esophagus. A transthoracic echocardiogram (TTE) was performed which demonstrated normal intracardiac anatomy and an RAA with an aberrant left subclavian artery (ALSA), suggestive of a VR. In order to confirm the diagnosis, CT angiogram (CTA) and cardiac magnetic resonance imaging (CMR) were considered. Due to concern for the need for sedation and possibly intubation in order to obtain a CMR in an infant with respiratory compromise, the decision was made to proceed with CTA. CTA demonstrated a RAA with retroesophageal ALSA arising from a Kommerell's diverticulum with tracheal and esophageal 

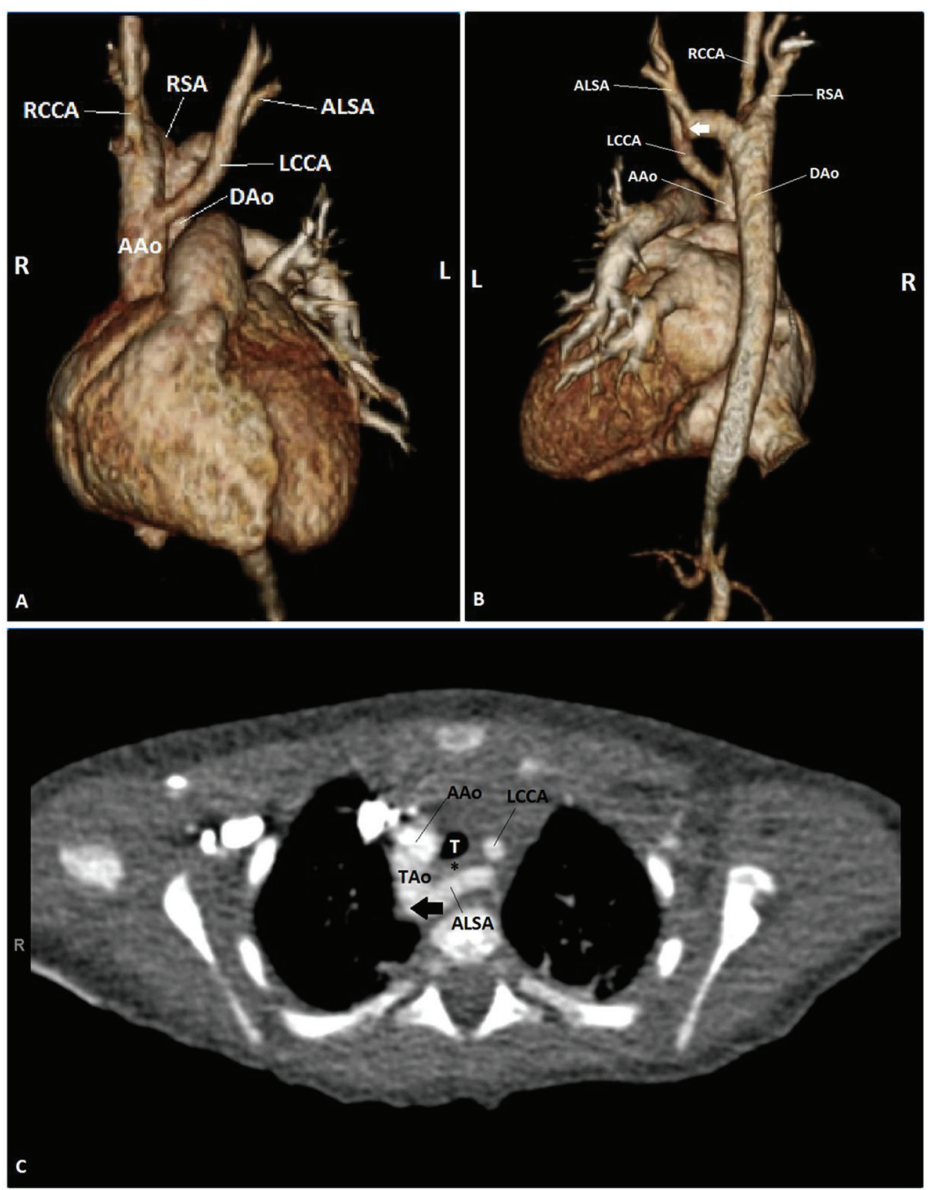

Figure 1. (A) Contrast-enhanced CT angiography three-dimensional multiplanar reformat, anterior view. Right-sided aortic arch with aberrant left subclavian artery (ALSA) coming off of Kommerell's diverticulum. (B) Contrast-enhanced CT angiography three-dimensional multiplanar reformat, posterior view. Right-sided aortic arch with aberrant left subclavian artery (ALSA) coming off of Kommerell's diverticulum (white arrow). (C) Contrast-enhanced CT angiograph transverse view. Right-sided aortic arch with ALSA coming off of Kommerell's diverticulum (black arrow) showing right lateral compression of trachea (T) and complete compression of the esophagus (black asterisk). AAo: ascending aorta; ALSA: aberrant left subclavian artery; DAo: descending aorta; LCCA: left common carotid artery; RCCA: right common carotid artery; RSA: right subclavian artery; TAo: transverse aorta; T: trachea.

compression (Fig. 1). The patient subsequently underwent division of the ligamentum arteriosum via left thoracotomy with no major post-operative complications and was discharged 6 days after his operation. He continues to follow with pediatric pulmonology for management of his $\mathrm{CF}$ and his choking, emesis and stridor have resolved.

\section{Discussion}

To our knowledge, there are no described associations between $\mathrm{CF}$ and congenital heart disease, including no associations with VR. This patient presented a diagnostic challenge, as his presentation at birth of prolonged respiratory distress was attributed to meconium aspiration, his subsequent symptoms of frequent cough and noisy breathing were initially attributed to his recently diagnosed $\mathrm{CF}$, and his emesis was thought to be normal physiological reflux seen commonly in neonates and infants.

A VR is an aortic arch anomaly where vascular structures completely surround the trachea and esophagus [4] with an incidence of 1 in 1,000 [5]. In the human embryo, six pairs of aortic arches develop at different stages of organogenesis, with the fourth primitive left aortic arch forming the adult aortic arch and the right fourth generally disappearing. This produces the normal course of the aorta arching to the left and descending left of the spine. Various arch anatomies can result depending on combinations of arch persistence and regression $[1,4$, $6]$.

Double aortic arch and RAA with ALSA, Kommerell's diverticulum and left ductal ligament are the two most common causes of complete VR [5]. VR can be asymptomatic, or present with symptoms of respiratory distress, stridor, wheezing, dyspnea, recurrent respiratory tract infections and dysphagia. Even more rarely, they can cause more atypical symptoms such as reflex apnea and cyanosis. These symptoms vary in 
severity, and can present anywhere from infancy to adulthood. Asymptomatic patients do not generally require surgery unless cardiac surgery is performed for other associated congenital heart disease. If symptomatic, then surgery is recommended $[3,4]$.

If a VR is suspected based on history and physical examination, TEE, barium esophagram, bronchoscopy and/or esophagoscopy can aid in diagnosis. Although there is a degree of variability in diagnostic approaches for diagnosing VR $[2,7]$, some advocate CTA or CMR to provide a detailed description of the anatomy of the anomalous vessels and their relationship with the trachea and the esophagus. In RAA with ALSA, CTA and CMR can not only define the aortic arch sidedness and branching pattern, but also can better define associated Kommerell's diverticulum and left ductal ligament which if present, complete the VR $[3,8,9]$.

\section{Conclusion}

VR can be difficult to diagnose, as the trachea-esophageal compressive symptoms can be insidious and often attributed to other etiologies. Diagnosis of VR requires a high index of suspicion. Known symptomatology should be elicited if VR is suspected, and demonstration of aortic arch anomalies on TTE suspicious for VR should be further investigated by CTA and CMR to fully define the aortic arch and branching anatomy in relationship to the trachea and esophagus.

\section{Financial Disclosure}

The authors have received no grants, honorariums or funding to write this manuscript.

\section{Conflict of Interest}

The authors hold no conflicts of interest.

\section{References}

1. Powell AJ, Mandell VS. Vascular rings and slings. In: Kean JF, Lock JE, Fyler DC, eds. Nadas' Pediatric Cardiology. 2nd ed. Philadelphia: Saunders Elsevier; 2006. p. 811-823.

2. Turner A, Gavel G, Coutts J. Vascular rings--presentation, investigation and outcome. Eur J Pediatr. 2005;164(5):266-270.

3. Kir M, Saylam GS, Karadas U, Yilmaz N, Cakmakci H, Uzuner N, Guzeloglu M, et al. Vascular rings: presentation, imaging strategies, treatment, and outcome. Pediatr Cardiol. 2012;33(4):607-617.

4. Weinberg PM, Natarajan S, Rogers LS. Aortic Arch and Vascular Anomalies. In: Allen HD, Driscoll DJ, Shaddy RE, Feltes TF, eds. Moss and Adams' Heart Disease in Infants, Children, and Adolescents: Including the Fetus and Young Adult. 8th ed. Philadelphia: Lippincott Williams \& Wilkins; 2013. p. 759-798.

5. Tuo G, Volpe P, Bava GL, Bondanza S, De Robertis V, Pongiglione G, Marasini M. Prenatal diagnosis and outcome of isolated vascular rings. Am J Cardiol. 2009;103(3):416-419.

6. Cina CS, Althani H, Pasenau J, Abouzahr L. Kommerell's diverticulum and right-sided aortic arch: a cohort study and review of the literature. J Vasc Surg. 2004;39(1):131139.

7. Woods RK, Sharp RJ, Holcomb GW, 3rd, Snyder CL, Lofland GK, Ashcraft KW, Holder TM. Vascular anomalies and tracheoesophageal compression: a single institution's 25-year experience. Ann Thorac Surg. 2001;72(2):434438; discussion 438-439.

8. Donato R, Lentini S, Di Bella G. Magnetic resonance angiography for diagnosis of right aortic arch with vascular ring. Arch Cardiovasc Dis. 2010;103(11-12):631-633.

9. Paparo F, Bacigalupo L, Melani E, Rollandi GA, Caro GD. Cardiac-MRI demonstration of the ligamentum arteriosum in a case of right aortic arch with aberrant left subclavian artery. World J Radiol. 2012;4(5):231-235. 